\title{
Effects of head motion on postural stability in healthy young adults with chronic motion sensitivity
}

\author{
Abdulaziz A. Albalwi ${ }^{1 *}$, Eric G. Johnson ${ }^{2}$, Ahmad A. Alharbi ${ }^{1}$, Noha S. Daher ${ }^{3}$, Tim K. Cordett ${ }^{2}$, \\ Oluwaseun I. Ambode ${ }^{2}$ and Fahad H. Alshehri ${ }^{2}$
}

\begin{abstract}
Background: Motion sensitivity, or motion sickness, is common in modern vehicular and visually stimulating environments. Several studies have shown a relationship between motion sensitivity and decreased postural stability. We aimed to evaluate the effects of head motion (horizontal and vertical) on postural stability in healthy adults with and without chronic motion sensitivity (CMS).

Methods: Sixty healthy adult men and women (age, 20-40 years) with CMS (CMS group, $n=30$ ) and without CMS (non-CMS group, $n=30$ ) participated in the study. Postural stability was assessed during three conditions (static, horizontal head motion, and vertical head motion) using computerized dynamic posturography. Group and condition-related differences in equilibrium scores were evaluated.

Results: There was no significant group $x$ condition interaction $\left(F_{2,114}=0.9\right.$, partial $\left.\eta^{2}=0.04, p=0.35\right)$. However, significant condition-related differences in equilibrium scores were observed $\left(F_{2,114}=26.4\right.$, partial $\eta^{2}=0.31, p<$ 0.001). Equilibrium scores were significantly worse in the horizontal and vertical head motion conditions compared to those in the static condition $(p<0.001)$, but were comparable in vertical and horizontal head motion conditions $(p=0.27)$.
\end{abstract}

Conclusions: Postural stability was lower in the horizontal and vertical conditions compared to the static condition. However, horizontal and vertical head motions had comparable effects on postural stability in both CMS and nonCMS groups, contrary to our expectations.

Keywords: Motion sensitivity, Postural stability, Equilibrium, Head motion

\section{Background}

Motion sensitivity, or motion sickness, is common in modern vehicular and visually stimulating environments, and individuals with normal vestibular function are susceptible [1]. It has been reported that $28.4 \%$ of the population experience motion sensitivity [2], and it is more common in women than in men $[2,3]$. Transportation,

\footnotetext{
* Correspondence: aa-albalwi@ut.edu.sa; abuaceil@hotmail.com

'Department of Physical Therapy, Faculty of Applied Medical Sciences, Tabuk University, Duba Road, Tabuk 71491, Saudi Arabia

Full list of author information is available at the end of the article
}

such as cars, trains, amusement park rides, airplanes, boats, and entertainment innovations (e.g., virtual reality), play a major role in increasing the prevalence of motion sensitivity [4]. Furthermore, transportation, in general, is a part of everyday life for most people [5].

Motion sensitivity is traditionally defined as the onset of nausea or vomiting experienced by individuals traveling by air, sea, space, and land, leading to impaired function [6]. Symptoms of motion sensitivity include visual and postural instability, pallor, sweating, excess salivation, headaches, drowsiness, malaise, nausea, and 
vomiting $[5,7]$. The most widely accepted mechanism is the sensory conflict theory [8], which states that motion sensitivity is the result of a sensory input mismatch (visual, vestibular, and proprioceptive) $[6,9,10]$. In other words, information provided by one sensory system does not match the expected input from another system; usually, a mismatch between the vestibular and visual systems is involved $[6,9,10]$. An alternative theory is the postural instability theory, which states that motion sensitivity is caused by a loss of postural control [11].

Postural stability is a complex task, requiring the proper integration of sensory inputs from visual, vestibular, and proprioceptive systems [12-14]. Therefore, postural stability includes the coordination of movement strategies to maintain the center of body mass during both self-initiated and externally triggered disturbances in balance [14]. Individuals with motion sensitivity often complain of postural instability and/or dizziness, which is a bothersome feeling that can be associated with head motion and not necessarily the result of vestibular dysfunction $[15,16]$. Stimulation of the vestibular system activates the vestibulo-ocular reflex and the vestibulospinal reflex, while stimulation of the upper neck-joint receptors activates the cervico-ocular reflex [17]. Consequently, both head and neck rotation contribute to stimulating these reflexes [18]. In addition, increased postural instability can be stimulated by either active head rotation or head tilt in patients with vestibular dysfunction $[19,20]$ as well as in healthy individuals [21, 22]. Finally, several studies have shown a relationship between motion sensitivity and postural instability [23-27]. For example, Owen et al. [27] found that greater postural instability was correlated with motion sensitivity. The authors reported that motion sensitivity susceptibility correlated most strongly with postural instability during conditions of visual and somatosensory feedback was absent or distorted.

Additional research supports an effect of head movement on postural stability. For example, Guedry and Benson [28] investigated Coriolis cross-coupling effects on healthy individuals and found that head movements can cause nausea and disorientation. Furthermore, head movements in weightlessness, especially in the pitch direction, are likely to cause motion sensitivity [29]. However, horizontal movements are more likely relevant to the routine activities of daily life and comprise a substantial portion of the head movements associated with daily balance activities [19]. Lackner and Graybiel [30] examined the effects of head movement direction (i.e., yaw, roll, and pitch) on motion sensitivity and found that all movements provoke sensitivity. In addition, Paloski et al. [21] examined the effects of different head movement frequencies on postural control in healthy individuals and found that postural instability was increased during dynamic head tilts. Thus, postural stability may be worse during vertical head motion compared to that during horizontal head motion.

Given the functional relationship between dynamic head motion and postural stability, people with chronic motion sensitivity (CMS) may experience additional challenges in their daily lives. To our knowledge, the effects of head motion on postural stability in individuals with CMS have not been reported. Therefore, we aimed to investigate the effects of head motion (horizontal, vertical) on postural stability in healthy adults with and without CMS. The primary hypothesis was that postural stability during head motion would be worse in the CMS group compared to that in the non-CMS group. The secondary hypothesis was that postural stability would be worse during vertical head motion compared to that during horizontal head motion for adults with or without CMS.

\section{Methods \\ Design}

The present study utilized an observational crosssectional design.

\section{Participants}

A total of 60 young adult participants aged from 20 to 40 years old from Loma Linda University and the local community (30 men and 30 women with a mean age of $26.8 \pm 4.3$ years and a body mass index [BMI] of $24.9 \pm$ $4.6 \mathrm{~kg} / \mathrm{m}^{2}$ ) were recruited for this study via email, word of mouth, and flyers posted around the university campus. Participants with a history of vestibular disorders, neurological pathology, head or cervical trauma, lack of a normal cervical spine active range of motion, Motion Sensitivity Susceptibility Questionnaire-Short Form (MSSQ-SF) [31] score between the 25th and 30th percentile, and those who were taking any medications that might affect balance were excluded from the study. Participants were divided into those with a self-reported history of CMS and an MSSQ-SF score in the 30th percentile or more (CMS group, $n=30$ ) and those without a self-reported history of CMS and a MSSQ-SF score in the 25th percentile or less (non-CMS group, $n=30$ ). The MSSQ-SF does not have a specific cut-off value; thus, the use of the 30th percentile was based on the recommendation of the author of the MSSQ-SF and the findings of a previous study that reported the 30th percentile as the lowest score in the CMS group [32]. We excluded participants who scored between the 25th and 30th percentile to create a "gap" between the two groups. This study was conducted at Loma Linda University in the Physical Therapy Neuroscience Research Laboratory. 


\section{Ethics}

All participants provided written informed consent, and the study was approved by the University Institutional Review Board.

\section{Procedures}

All participants completed the MSSQ-SF, which was designed to assess the types of motion that cause motion sickness in children and adults [31]. The MSSQ-SF has a high correlation with the MSSQ-Long Form $(r=0.93)$. In addition, the MSSQ-SF exhibits high internal consistency (Cronbach's alpha $=0.87$ ), test-retest reliability $(r=0.9)$, and a significant correlation between Section A (Child) and Section B (Adult) results $(r=0.68)$ [31].

In addition, participants self-reported their physical activity level and anthropometric measurements (weight and height) were taken. Participants were then trained on the specific parameters of cervical rotation, flexion, and extension. To prevent falling, participants donned a safety harness and two investigators stood behind the participant during all postural stability testing. Postural stability was measured during three conditions (static, horizontal head motion, and vertical head motion) using a computerized dynamic posturography (CDP) forceplate system (Bertec Balance Advantage Dynamic CDP, Bertec Corporation, Columbus, OH, USA). Each condition included three twenty-second trials; the mean of the three trials was analyzed.

Participants performed all conditions while standing on a CDP force plate with bare feet. For dynamic conditions (head motions), the head velocity and amplitude was selected based on slow-speed walking, as this is a common physical activity of daily living for most individuals [33]. Based on the reported normal head velocity and amplitude during walking, a velocity of $1.5 \mathrm{~Hz}$ [34], and head motion amplitudes of 11 degrees in the horizontal direction and 8 degrees in the vertical direction [35] were utilized. The dynamic conditions were measured with the participants performing active head motions (horizontal or vertical), in randomized order, moving their heads to the auditory cue of a metronome set at $1.5 \mathrm{~Hz}$.

In contrast to several previous studies, in which participants performed head movements with their eyes closed during sensory organization testing by holding their hands 15 degrees to each side of their face to control the range of motion $[19,20,36]$, the present study utilized a head-mounted laser pointer (SenMoCOR LED/Laser, Orthopedic Physical Therapy Products, USA). Participants were instructed to keep their eyes open to guide the range of motion amplitude, which allowed the use of the amplitude and velocity of head motion during slow walking as a reference. Furthermore, people usually keep their eyes open during locomotion to explore the environment around them. Additionally, a grid was positioned at the same height as the participant's eyes, $90 \mathrm{~cm}$ from the participant's forehead, to aid in tracking the laser (Fig. 1). Under the guidance of the laser pointer and verbal cues from the investigators, the participants maintained a range of motion amplitude of approximately 11 degrees in the horizontal plane (5.5 degrees to each side) and 8 degrees in the vertical plane (4 degrees up and 4 degrees down).

Participants postural stability, or equilibrium score, was calculated using the following formula: Equilibrium Score $(E S)=(12.5-($ Max-AP COG Angle $)) / 12.5$ X 100.

The equation for sway angle is: Sway Angle CDP = arc$\sin (\mathrm{COGy} /(.55 \% \mathrm{~h}))$ where $\mathrm{y}=$ anterior-posterior sway axis and $\mathrm{h}=$ the subject's height in [ $\mathrm{cm}$ or inches]. The inverse $\mathrm{Sin}$ of the center of gravity was divided by $55 \%$ of the subject's height. Subjects exhibiting little sway achieve equilibrium scores near 100, while subjects whose sway approaches their limits of stability achieve scores near zero. The equilibrium score ranges between 0 and 100\%; higher values reflect better postural stability [37].

\section{Statistical analysis}

A sample size of 60 participants was estimated using GPower software (version 3.1.2, University of Dusseldorf, Dusseldorf, Germany) assuming a medium effect size of $(f=0.25)$, a power of 0.80 , and a level of significance $(\alpha)$ of 0.05. Data analyses were performed using the SPSS statistical package for Windows, version 22.0 (SPSS, Inc., Chicago, IL). Descriptive statistics are given as mean and standard deviation for quantitative variables, and as frequency and percentage (\%) for categorical variables. Group differences in the frequency distribution of sex and physical activity level were evaluated using chisquare tests. Normality was assessed using the Kolmogorov-Smirnov test. Group differences in height, weight, and body mass index (BMI) were evaluated using the independent-sample $t$-test. Since significant group differences in age were observed, group and conditionrelated changes in equilibrium scores (static vs. horizontal vs. vertical) were examined using a mixed factorial analysis of variance (ANOVA), after controlling for age. Post hoc analyses with Bonferroni correction were conducted. The level of significance was set at $p \leq 0.05$.

\section{Results}

The 60 participants had a mean age of $26.8 \pm 4.3$ years and mean body mass index (BMI) of $24.9 \pm 4.6\left(\mathrm{~kg} / \mathrm{m}^{2}\right)$. There were no significant group differences in mean height $(\mathrm{m})$, weight $(\mathrm{kg})$, or BMI $\left(\mathrm{kg} / \mathrm{m}^{2}\right) \quad(p>0.05$, Table 1). In addition, the groups did not differ in sex distribution or physical activity level (Table 1). However, 


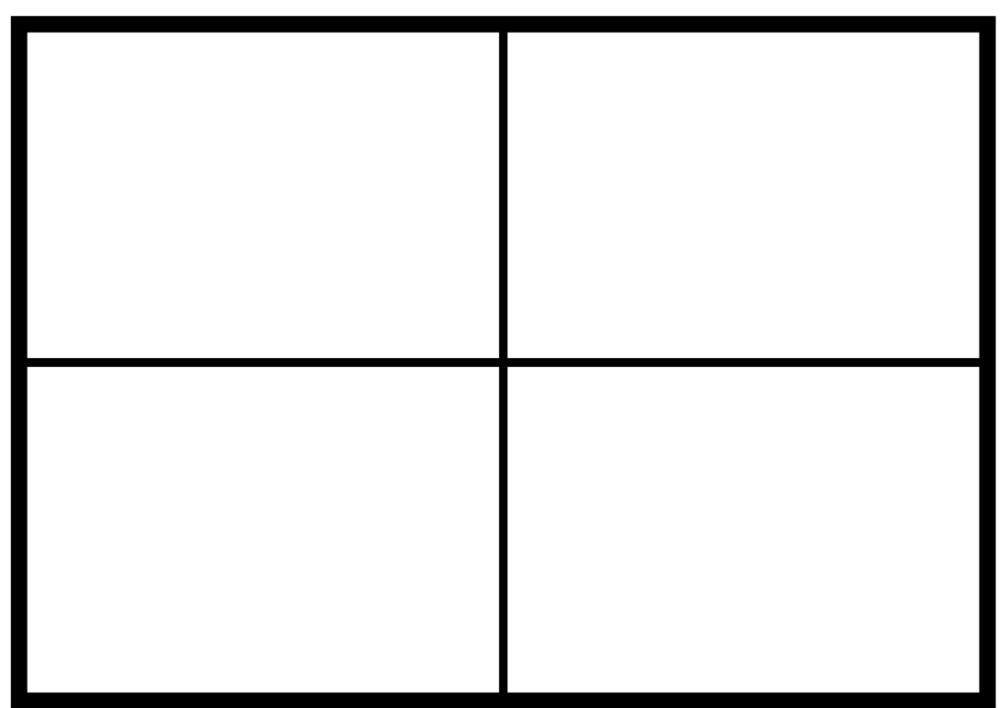

Fig. 1 Grid to guide the amplitude of horizontal $\left(11^{\circ}\right)$ and vertical $\left(8^{\circ}\right)$ head motions

there was a significant difference in mean age between the two groups $(27.9 \pm 4.5$ vs. $25.6 \pm 3.8, p=0.04$, Table $1)$. In addition, there was no significant difference in mean equilibrium score during static condition by group $(93.8 \pm 2.7$ vs. $94.9 \pm 1.3, p=0.25)$.

Results of the mixed factorial ANOVA are displayed in Table 2. There was no significant group by condition interaction $\left(\mathrm{F}_{2,114}=0.9\right.$, partial $\left.\eta^{2}=0.04, p=0.35\right)$. However, the mean equilibrium score differed significantly among the three tested conditions $\left(F_{2,114}=26.4\right.$, partial $\left.\eta^{2}=0.31, p<0.001\right)$. Post hoc comparisons using Bonferroni adjustment revealed that mean \pm standard error equilibrium scores were significantly different between horizontal head motion and static condition $(92.4 \pm 0.4$

Table 1 General baseline characteristics of the study participants $(N=60)$

\begin{tabular}{lllc}
\hline Characteristic & CMS $\left(n_{1}=30\right)$ & Non-CMS $\left(n_{2}=30\right)$ & $p$-value \\
\hline Gender; $n$ (\%) & & & 0.22 \\
$\quad$ Female & $13(43.3)$ & $17(56.7)$ & \\
$\quad$ Male & $17(56.7)$ & $13(43.3)$ & 0.04 \\
Age (years) & $27.9(4.5)$ & $25.6(3.8)$ & 0.62 \\
Height (m) & $1.7(0.1)$ & $1.7(0.1)$ & 0.17 \\
Weight (kg) & $75.1(20.6)$ & $68.7(14.6)$ & 0.14 \\
BMI (kg/m $\left.{ }^{2}\right)$ & $25.8(5.6)$ & $24.1(3.2)$ & 0.29 \\
Physical Activity; $\mathrm{n}(\%)$ & & & \\
Often & $11(36.7)$ & $14(46.7)$ & \\
Sometimes & $16(53.3)$ & $15(50.0)$ & \\
Never & $3(10.0)$ & $1(3.3)$ & \\
\hline
\end{tabular}

Abbreviations: SD Standard Deviation; CMS Chronic Motion Sensitivity; BMI Body Mass Index vs. $94.4 \pm 0.3, p<0.001)$ and vertical head motion condition compared to the static condition $(91.9 \pm 0.5$ vs. $94.4 \pm 0.3, p<0.001)$, however, there was no significant difference between horizontal and vertical conditions $(p=0.27)$. Similar results were obtained after adjusting for age.

\section{Discussion}

Building upon the work of Owen et al. [27], the present study describes the effects of head motion on postural stability in healthy adults with and without CMS. The results demonstrate that postural stability differs by the condition of head motion. Postural stability was lower in the horizontal and vertical conditions compared to the static condition. However, horizontal and vertical head motions had comparable effects on postural stability in both CMS and non-CMS individuals, contrary to our expectations. Consistent with the present results, Mitsutake et al. [38] reported that postural stability during active horizontal head motion (during eyes open and closed conditions) was significantly decreased in stroke patients compared to that in healthy people. In addition, Paloski et al. [21] reported that healthy subjects were able to maintain an upright stance during static head tilts with eyes closed; however, postural stability was decreased during dynamic head tilts with eyes closed, especially with higher degrees of head tilt.

Many studies have measured unperturbed body sway before participants were exposed to visual motion stimuli [23-26]. The results from these studies demonstrate that pre-exposure postural stability is diminished in participants who became sick after motion exposure compared to that in those who did not become motion sick, consistent with the present results. Furthermore, a study 
Table 2 Mean (SE) equilibrium scores during head motion by group $(N=60)$

\begin{tabular}{lllc}
\hline Condition $^{a}$ & CMS $\left(n_{1}=30\right)$ & Non-CMS $\left(n_{2}=30\right)$ & Group x condition $p$-value ${ }^{*}\left(\right.$ partial $\left.\eta^{2}\right)$ \\
\hline Static condition & $93.8(0.3)$ & $94.9(0.4)$ & $0.11(0.04)$ \\
Horizontal & $91.1(0.6)$ & $93.6(0.6)$ & \\
Vertical & $90.7(0.7)$ & $93.1(0.7)$ & \\
\hline
\end{tabular}

Abbreviations: SE Standard Error; CMS Chronic Motion Sensitivity

${ }^{a}$ Significant difference between static and horizontal, and static and vertical conditions $(p<0.001)$

${ }^{*}$ Mixed factorial ANOVA

by Stoffregen, Chen, and Koslucher [39] found that movement of the head and torso differed between participants who later became motion sick and those who did not. Together, these studies provide support for the postural instability theory. Owen et al. [27] reported that greater postural instability was correlated with motion sensitivity during conditions of visual and somatosensory feedback absence or distortion. The results of the present study do not support these assumptions; however, the conditions of postural stability in this investigation did not remove or distort visual or somatosensory input.

Sensory systems (visual, somatosensory, and vestibular), central processing, musculoskeletal systems, and neural pathways are essential for postural stability [40, 41]. To maintain postural stability, the vestibular system provides the central nervous system with information about head motion relative to space [42]. This information estimates the orientation of an individual in space and the degree of tilt from gravity vertical, which assists the individual in maintaining upright while standing and walking [42]. Paillard et al. [43] indicated that the vestibular system might be involved in CMS. Vestibular system involvement in CMS, and the stimulation of the vestibular system during head motion, may explain the observed reduced postural stability during head motion in the CMS group compared to that in the non-CMS group.

We also examined whether horizontal and vertical head movements differentially impact postural stability in adults with and without CMS. The results suggest that postural stability is similar in the CMS group compared to that in the non-CMS group for both horizontal and vertical head movements during conditions of normal vision and slow velocity head motion. Functional implications of horizontal and vertical head motions include a variety of standing activities (e.g., checking for traffic before crossing the street, looking in kitchen cabinets, and showering).

Horizontal movements are likely more relevant to routine activities of daily life and comprise a fundamental portion of head movements associated with daily balance activities [19]. In addition, horizontal eye and head motions are often utilized to guide changes in walking directions [44]. Consequently, we hypothesized that postural stability would be worse during vertical head motion compared to that during horizontal head motion within both groups; however, this hypothesis was not supported. Lackner and Graybiel [30] demonstrated that all movements (yaw, roll, and pitch) provoked motion sensitivity; however, pitch head movements (vertical motion) were the most stressful. In other investigations, individuals with vestibular disorders reported more falls while walking with vertical head movement than while walking with horizontal head movement [45]. However, others have reported that no difference exists in walking speed during horizontal and vertical head movements [46]. In the present study, the amplitude of the horizontal head motion was greater compared to that for the vertical head motion. Thus, we speculate that the difference in the amplitude of the head range of motions (11 degrees horizontal versus 8 degrees vertical) may explain the lack of significant differences between the head motion conditions.

Although the design of the present study included potential confounders (i.e., the visual cues during the laserguided head motion and auditory attention to the metronome guiding head motion velocity), a previous study indicated that a secondary task, such as an auditory signal, does not affect balance control [21]. In addition, the performance of simultaneous tasks, such as walking while talking with friends and watching the world around us, is necessary during daily activities. Therefore, motor and cognitive tasks do not always require conscious attention and can be performed automatically [47]. Further study is needed to investigate the effects of dual-task performance on postural stability in individuals with CMS.

\section{Limitations}

The present study had several limitations. The main limitation concerns the narrow age range of the participants (20-40 years of age); thus, the findings may not be generalizable to older adults. Also, a valid and reliable physical activity questionnaire was not utilized, and inactivity can affect postural stability [48]. Finally, vestibular function testing was not performed. Future studies should include additional age ranges, perform vestibular function testing, consider varying the head motion velocity and amplitude, and investigate whether standing 
gaze-stability exercises [32, 49] can improve postural stability.

\section{Conclusions}

The results demonstrate that postural stability differs by the condition of head motion. Postural stability was lower in the horizontal and vertical conditions compared to the static condition. However, horizontal and vertical head motions had comparable effects on postural stability in both CMS and non-CMS groups, contrary to our expectations.

\section{Abbreviations}

ANOVA: Analysis of variance; BMI: Body mass index; CDP: Computerized dynamic posturography; CMS: Chronic motion sensitivity; MSSQ-SF: Motion Sensitivity Susceptibility Questionnaire-Short Form; SD: Standard Deviation; SE: Standard Error

\section{Acknowledgements}

The authors thank Loma Linda University, Tabuk University, and the Saudi Arabian Cultural Mission for supporting this research. We also thank Intithar Elias for providing technical support and graduate student, Parita Shah, for assisting in the research process.

\section{Authors' contributions}

AA Albalwi completed the research work as this was his thesis project for DSc in Physical Therapy. EGJ served as supervisor and content expert. TCN was a research committee member and additional content expert. NSD was a research committee member and did the statistical analysis of data. AA Alharbi, OIA, and FHA were co-investigators on this research. AA Albalwi reported the results of this research as an oral defense for DSc in Physical Therapy at Loma Linda University in March 2017. All authors read and approved the final manuscript.

\section{Funding}

This research was supported by the Department of Physical Therapy at Loma Linda University, CA, USA and Tabuk University, Tabuk, KSA.

\section{Availability of data and materials}

The datasets used and/or analysed during the current study are available from the corresponding author on reasonable request.

\section{Ethics approval and consent to participate}

The study was approved by the Loma Linda University Institutional Review Board (Approval number: 5160219). All participants provided written informed consent.

\section{Consent for publication}

Consent for publication was obtained from the participants.

\section{Competing interests}

The authors declare that they have no competing interests.

\section{Author details}

'Department of Physical Therapy, Faculty of Applied Medical Sciences, Tabuk University, Duba Road, Tabuk 71491, Saudi Arabia. ${ }^{2}$ Department of Physical Therapy, School of Allied Health Professions, Loma Linda University, Loma Linda, CA, USA. ${ }^{3}$ Department of Allied Health Studies, School of Allied Health Professions, Loma Linda University, Loma Linda, CA, USA.

Received: 24 August 2018 Accepted: 20 March 2020 Published online: 30 March 2020

\section{References}

1. Bronstein AM, Golding JF, Gresty MA. Vertigo and dizziness from environmental motion: visual vertigo, motion sickness, and drivers disorientation. Semin Neurol. 2013;33(3):219-30.
2. Turner M, Griffin MJ. Motion sickness in public road transport: passenger behavior and susceptibility. Ergonomics. 1999:42(3):444-61.

3. Koslucher F, Haaland E, Malsch A, Webeler J, Stoffregen TA. Sex differences in the incidence of motion sickness induced by linear visual oscillation. Aerosp Med Hum Perform. 2015;86(9):787-93.

4. Golding JF. Motion sickness susceptibility. Auton Neurosci. 2006;129(1-2): $67-76$.

5. Murdin L, Golding J, Bronstein A. Managing motion sickness. BMJ. 2011;343: d7430.

6. Reason JT, Brand JJ. Motion sickness. New York: Academic Press; 1975 p. 38-81.

7. Rine RM, Schubert MC, Balkany TJ. Visual-vestibular habituation and balance training for motion sickness. Phys Ther. 1999:79(10):949-57.

8. Z Zhang LL, Wang JQ, Qi RR, Pan LL, Li M, Cai YL. Motion sickness: current knowledge and recent advance. CNS Neurosci Ther. 2016;22(1):15-24.

9. Reason JT. Motion sickness adaptation: a neural mismatch model. J R Soc Med. 1978;71(11):819-929.

10. Macefield VG, Walton DK. Susceptibility to motion sickness is not increased following spinal cord injury. J Vestib Res. 2015;25(1):35-9.

11. Riccio GE, Stoffregen TA. An ecological theory of motion sickness and postural instability. Ecol Psychol. 1991;3(3):195-240.

12. Pang MY, Lam FM, Wong GH, Au H, Chow DL. Balance performance in head-shake computerized dynamic posturography: aging effects and testretest reliability. Phys Ther. 2011;91(2):246-53.

13. Shaffer SW, Harrison AL. Aging of the somatosensory system: a translational perspective. Phys Ther. 2007;87(2):193-207.

14. Horak FB. Postural orientation and equilibrium: what do we need to know about neural control of balance to prevent falls? Age Ageing. 2006;35: ii7-ii11.

15. Akin FW, Davenport MJ. Validity and reliability of the motion sensitivity test. J Rehabil Res Dev. 2003;40(5):415-21.

16. Norré ME, Beckers A. Vestibular habituation training for positional vertigo in elderly patients. Arch Gerontol Geriatr. 1989;8(2):117-22.

17. Hikosaka O, Maeda M. Cervical effects on abducens motoneurons and their interaction with vestibulo-ocular reflex. Exp Brain Res. 1973;18(5):512-30.

18. Padoan S, Karlberg M, Fransson PA, Magnusson M. Passive sustained turning of the head induces asymmetric gain of the vestibulo-ocular reflex in healthy subjects. Acta Otolaryngol. 1998;118(6):778-82.

19. Mishra A, Davis S, Speers R, Shepard NT. Head shake computerized dynamic posturography in peripheral vestibular lesions. Am J Audiol. 2009;18(1):53-9.

20. Honaker JA, Converse CM, Shepard NT. Modified head shake computerized dynamic posturography. Am J Audiol. 2009;18(2):108-13.

21. Paloski WH, Wood SJ, Feiveson AH, Black FO, Hwang EY, Reschke MF. Destabilization of human balance control by static and dynamic head tilts. Gait Posture. 2006:23(3):315-23.

22. Morimoto $H$, Asai $Y$, Johnson EG, Lohman EB, Khoo K, Mizutani $Y$, et al. Effect of oculo-motor and gaze stability exercises on postural stability and dynamic visual acuity in healthy young adults. Gait Posture. 2011 33(4):600-3

23. Stoffregen TA, Smart $L J$ Jr. Postural instability precedes motion sickness. Brain Res Bull. 1998:47(5):437-48.

24. Smart $L J \mathrm{Jr}$, Stoffregen TA, Bardy BG. Visually induced motion sickness predicted by postural instability. Hum Factors. 2002:44(3):451-65.

25. Stoffregen TA, Yoshida K, Villard S, Scibora L, Bardy BG. Stance width influences postural stability and motion sickness. Ecol Psychol. 2010;22(3): 169-91.

26. Villard SJ, Flanagan MB, Albanese GM, Stoffregen TA. Postural instability and motion sickness in a virtual moving room. Hum Factors. 2008;50(2):332-45.

27. Owen N, Leadbetter AG, Yardley L. Relationship between postural control and motion sickness in healthy subjects. Brain Res Bull. 1998;47(5):471-4.

28. Guedry FE Jr, Benson AJ. Coriolis cross-coupling effects: disorienting and nauseogenic or not? Aviat Space Environ Med. 1978:49:29-35.

29. Oman CM, Lichtenberg BK, Money KE, McCoy RK. M.IT./Canadian vestibular experiments on the Spacelab-1 mission: 4. Space motion sickness: symptoms, stimuli, and predictability. Exp Brain Res. 1986;64(2):316-34

30. Lackner JR, Graybiel A. Head movements elicit motion sickness during exposure to microgravity and macrogravity acceleration levels. In: Igarashi $\mathrm{M}$, Black FO, editors. Vestibular and visual control on posture and locomotor equilibrium. New York: Karger; 1985. p. 170-7.

31. Golding JF. Predicting individual differences in motion sickness susceptibility by questionnaire. Pers Individ Dif. 2006;41(2):237-48. 
32. Gaikwad SB, Johnson EG, Nelson TC, Ambode Ol, Albalwi AA, Alharbi AA, et al. Effect of gaze stability exercises on chronic motion sensitivity. J Neurol Phys Ther. 2018:42(2):72-9.

33. Brooks GA, Butte NF, Rand WM, Flatt JP, Caballero B. Chronicle of the Institute of medicine physical activity recommendation: how a physical activity recommendation came to be among dietary recommendations. Am J Clin Nutr. 2004;79(5):921S-30S.

34. Hirasaki E, Moore ST, Raphan T, Cohen B. Effects of walking velocity on vertical head and body movements during locomotion. Exp Brain Res. 1999; 127(2):117-30.

35. Bible JE, Biswas $\mathrm{D}$, Miller $\mathrm{CP}$, Whang $\mathrm{PG}$, Grauer JN. Normal functional range of motion of the cervical spine during 15 activities of daily living. J Spinal Disord Tech. 2010;23(1):15-21.

36. Moussa AM, Tawfik S, El Kholi W, Al-Haggagy M. Head shake posturography in peripheral vestibular disorders. Mediterr J Otol. 2008:4:9-18.

37. Bertec Balance Advantage user manual; 2014. http://bertec.com/ bertecbalance/. Accessed 18 Mar 2016.

38. Mitsutake T, Chuda Y, Oka S, Hirata H, Matsuo T, Horikawa E. The control of postural stability during standing is decreased in stroke patients during active head rotation. J Phys Ther Sci. 2014;26(11):1799-801.

39. Stoffregen TA, Chen YC, Koslucher FC. Motion control, motion sickness, and the postural dynamics of mobile devices. Exp Brain Res. 2014;232(4): 1389-97.

40. Viel S, Vaugoyeau M, Assaiante C. Adolescence: a transient period of proprioceptive neglect in sensory integration of postural control. Mot Contr. 2009;13(1):25-42.

41. Mallau S, Vaugoyeau M, Assaiante C. Postural strategies and sensory integration: no turning point between childhood and adolescence. PLoS One. 2010;5(9):e13078.

42. Sienko KH, Whitney SL, Carender WJ, Wall C. The role of sensory augmentation for people with vestibular deficits: real-time balance aid and/ or rehabilitation device? J Vestib Res. 2017;27(1):63-76.

43. Paillard AC, Quarck G, Paolino F, Denise P, Paolino M, Golding JF, et al. Motion sickness susceptibility in healthy subjects and vestibular patients: effects of gender, age, and trait-anxiety. J Vestib Res. 2013;23(4-5):203-9.

44. Glaister BC, Bernatz GC, Klute GK, Orendurff MS. Video task analysis of turning during activities of daily living. Gait Posture. 2007;25(2):289-94.

45. Whitney SL, Marchetti GF, Schade A, Wrisley DM. The sensitivity and specificity of the timed "up \& go" and the dynamic gait index for selfreported falls in persons with vestibular disorders. J Vestib Res. 2004;14(5): 397-409.

46. Schmidheiny A, Swanenburg J, Straumann D, de Bruin ED, Knols RH. Discriminant validity and test re-test reproducibility of a gait assessment in patients with vestibular dysfunction. BMC Ear Nose Throat Disord. 2015;15:6.

47. Santos LAA, Campos C, Bento T, Lattari E, Nardi AE, Rocha NBF, et al. Effects of dual-task interventions on gait performance of patients with Parkinson's disease: a systematic review. Med Express. 2016;3(4):M160401.

48. Hindmarsh JJ, Estes EH Jr. Falls in older persons: causes and interventions. Arch Intern Med. 1989;149(10):2217-22.

49. Alyahya D, Johnson EG, Daher NS, Gaikwad SB, Deshpande S, Cordett TK et al. Effect of vestibular adaptation exercises on chronic motion sensitivity: a randomized controlled trial. Phys Ther Rehabil. 2016;3(1):1.

\section{Publisher's Note}

Springer Nature remains neutral with regard to jurisdictional claims in published maps and institutional affiliations.

Ready to submit your research? Choose BMC and benefit from:

- fast, convenient online submission

- thorough peer review by experienced researchers in your field

- rapid publication on acceptance

- support for research data, including large and complex data types

- gold Open Access which fosters wider collaboration and increased citations

- maximum visibility for your research: over $100 \mathrm{M}$ website views per year

At BMC, research is always in progress.

Learn more biomedcentral.com/submissions 